\title{
Features Derived From Blood Pressure Predict Elevated Intracranial Pressure in Critically III Children
}

\section{Kassi Ackerman}

University of Tennessee Health Science Center

\section{Akram Mohammed}

University of Tennessee Health Science Center

\section{Lokesh Chinthala}

University of Tennessee Health Science Center

Robert L. Davis

University of Tennessee Health Science Center

\section{Rishikesan Kamaleswaran}

Emory University School of Medicine

Nadeem I. Shafi ( $\square$ nshafi@uthsc.edu )

University of Tennessee Health Science Center

\section{Research Article}

Keywords: eICP, ICP-derived features, ICP, AUROC, hemodynamic signals

Posted Date: July 26th, 2021

DOI: https://doi.org/10.21203/rs.3.rs-737405/v1

License: (1) This work is licensed under a Creative Commons Attribution 4.0 International License. Read Full License 


\section{Abstract}

Clinicians frequently observe hemodynamic changes preceding elevated intracranial pressure (ICP) events. We employed a machine learning approach to identify novel and differentially expressed features associated with elevated ICP (eICP) events in children with severe brain injuries. Statistical features from physiologic data streams were derived from non-overlapping 30-minute analysis windows prior to 21 elCP events; 200 records without elCP events were used as controls. Ten Monte Carlo simulations with training/testing splits provided performance benchmarks for 4 machine learning approaches. XGB yielded the best performing predictive models. SHAP analyses demonstrated that a majority of the top 20 contributing features from each simulation consistently derived from blood pressure data streams up to 240 minutes prior to elCP events, rivaling ICP-derived features at 0-60 minutes. Our AUROC benchmark at the 30-60 minutes analysis window using the XGB model bundle was 0.82 (95\% $\mathrm{Cl} 0.81-0.83$ ); the AUPRC was 0.24 (95\% $\mathrm{Cl} 0.23-0.25)$, well-above the expected baseline. We conclude that physiomarkers discernable by machine learning are concentrated within blood pressure data up to 4 hours prior to elCP events and demonstrate robust benchmark performance. Future predictive modeling of elevated ICP events should leverage features contained within hemodynamic signals.

\section{Introduction}

Elevations in intracranial pressure (ICP) begin to cause brain injury when cerebral blood vessels are compressed and neuronal function is compromised. The stereotyped vital sign changes that occur in response - hypertension, bradycardia, and irregular respirations - have come to be known as the Cushing reflex after being described in experimental animal models by Harvey Cushing in 1901 and 1902 [1]. This reflex is a late finding in elevated ICP states indicative of impending herniation. Often, tachycardia precedes the bradycardia due to an earlier sympathetic surge ${ }^{[2]}$.

The importance of identifying elevations in ICP early was highlighted by Stein et al., who conducted a prospective study in adult patients with severe traumatic brain injury (TBI) and concluded that the number of brief 5-minute episodes of cerebral hypoperfusion and intracranial hypertension was predictive of poor outcome ${ }^{[3]}$. Another study by Ferguson et al. showed that for every hour of ICP $>20$, the odds of poor outcomes increased by $4.6 \%{ }^{[4]}$. Accordingly, control of intracranial pressure and maintenance of cerebral perfusion are two overlapping pillars of brain injury management ${ }^{[5,6]}$.

It is natural, then, that clinicians and scientists would expect features contained within hemodynamic signals to offer early insights into elevated ICP (eICP) events. The application of machine-learning to physiologic signals offers an opportunity to detect higher-order features (i.e. those not discernable by human care providers) which may be predictive of clinical events. For instance, a machine learning model to predict increased ICP events in TBI patients was developed ${ }^{[7]}$ and externally validated ${ }^{[8]}$ using ICP and mean arterial pressure (MAP) as the primary model inputs. When differentially expressed, predictive features are sometimes referred to as 'physiomarkers.' [9-11]. 
Traditional approaches to machine learning often involve unbounded feature extraction that enlarges the dimensionality of the data followed by a subsequent reduction to a smaller set of relevant features ${ }^{[12]}$. This process can result in the generation of a significant number of features, often derived from the vast clinical data available within the electronic medical record and resulting in a complex black-box solution [13]. While this approach to developing models may reveal some meaningful performance benchmarks across single datasets, there are a number of problems of interpretability and generalization when such approach is applied externally ${ }^{[14,15]}$. Therefore, recent approaches have focused on the use of features localized by time and domain in order to reduce complexity and optimize 'attention' [16].

Alternatively, novel sources of features may be used, such as physiomarkers derived from streaming data that provide targeted views of specific aspects of human conditions ${ }^{[17]}$. Given that hemodynamic changes are clinically discernable before impending herniation, and in an effort to guide future modeling of elCP events, we analyzed continuous streaming data collected from bedside monitors using machine learning methods to evaluate (a) whether there are novel and differentially expressed physiomarkers of elCP events contained within hemodynamic and other physiologic signals, and (b) if these physiomarkers can reveal robust predictive performance.

\section{Methods}

\section{Clinical framework and data pre-processing}

We conducted a retrospective study of children aged 2-17 years who were admitted to the Pediatric ICU and Neuro ICU of a tertiary/quaternary children's medical center from October, 2017 until December, 2020. This study was approved by the Institutional Review Board of the University of Tennessee Health Science Center (Memphis, TN). All methods were performed in accordance with relevant guidelines and regulations as stipulated by the IRB, which waved the need for parent/caregiver consent for this study. To be included, children had to have undergone concurrent invasive arterial blood pressure (ABP) and ICP monitoring. Records which had $<4$ hours of concurrent electrocardiogram (EKG), ABP and ICP data were excluded.

All patients were managed in accordance with standards of care for brain injury, including the pediatric TBI guidelines ${ }^{5}$. An elevated ICP (eICP) event was defined as $\geq 20 \mathrm{~cm} \mathrm{H}_{2} \mathrm{O}$ for 10 minutes, and events were considered independent if they occurred at least 5 hours apart in the same patient (Fig. 1). Control records were derived from patients without elevated ICPs as well as from those with cases when ICPs were $<20 \mathrm{~cm} \mathrm{H}_{2} \mathrm{O}$ for 6 hours.

Six physiologic signals (ABP, consisting of systolic, diastolic, and mean blood pressures; electrocardiogram (EKG)-derived heart rate (HR); pulse rate from plethysmography; ICP; cerebral perfusion pressure (CPP); and oxygen saturation) were each sampled at 1-minute intervals via a Drager Monitoring system, with the view that employing lower resolution data would make the models translatable to a greater number of ICUs. 
All records were manually adjudicated by an ICU physician to confirm criteria and identify signal artifact. Records with $>10 \%$ missing data or significant artifact burden were excluded. Next, line graphs of the data were reviewed to manually identify obvious blood pressure artifact. These were removed with a twostep artifact detection algorithm after the standard deviation (SD) of the entire 4-hour record was determined. Because artifacts were most dramatically represented in the systolic blood pressure (SBP) data stream, any SBP value which exceeded the first datapoint of a 5-minute block by two SDs was removed and forward-filled; this was repeated after sliding the 5-minute block forwards by 1 -minute through the entire record. This step allowed us to identify and remove "wide artifact". Next, any SBP minute-to-minute change that exceeded $30 \%$ was removed and forward-filled, allowing identification and removal of "narrow artifact" that was not captured in the first step. At the points of artifact, SBP, MAP, DBP, and CPP data streams were all corrected. An example of artifact removal is provided in supplementary figures.

\section{Machine learning}

4-hour records were divided into 8 non-overlapping but consecutive 30 -minute analysis windows as depicted in Fig. 1. Prediction horizons - that is, the times in advance each model bundle was asked to predict the elCP event - were increased by 30 minutes until the beginning of the record. Eighteen statistical features were derived from the 30-minute window immediately prior to each prediction horizon. These were mean, median, max, min, quantiles $(0.2,0.4,0.6,0.8)$, variance, standard deviation, aggregated variance, centroid, kurtosis, skew, sample entropy, binned entropy, absolute change, and mean change.

Predictive modeling was performed with logistic regression (LR), support vector machine (SVM), random forest (RF), and XGBoost (XGB). The records were divided 70/30 for training and testing, respectively. Prior to training a model, we used a subset of the training data for hyperparameter selection using Bayesian optimization. ${ }^{[18]}$ Due to a significant imbalance between the numbers of cases (records with elCP events) and controls (records without elCP events), one control was chosen for each case in a random fashion during training. Training and testing were iterated with ten different 70/30 splits at each time window following 10 Monte Carlo simulations. Explainability of models generated were assessed using averaged Shapley Additive Explanations (SHAP) scores from each simulation. ${ }^{[19]}$ Model performance was assessed not only by area under the receiver operating characteristic (AUROC) curves, but also by area under the precision-recall (AUPRC) curves, which are more appropriate for imbalanced data sets. Sensitivity, specificity, positive and negative predictive values were also determined. An illustration of the architecture can be found in supplementary figures.

\section{Results}

\section{Cases, controls, and data preprocessing}


112 patients were identified using hospital procedure records for external ventricular drains and intraparenchymal pressure monitoring devices placed at our institution. 57 of the 112 patients met exclusion criteria and 55 patients met inclusion criteria. Of the 55 patients, 33 had complete datasets. Reasons for missingness included patients being transported off the unit, line or sensor dysfunction, etc. 24 patients had quality data with limited artifact burden that satisfied definitions for elCP events, controls, or both. A total of 21 cases from 9 patients and 200 controls from 22 patients were ultimately included for model development and testing (Fig. 2).

Our algorithmic artifact removal resulted in $1.2 \%$ overall data replacement (means of 2.8 and 2.8 minutes replaced per 4-hour record in cases and controls, respectively). $92 \%$ of the manually identified artifacts were removed.

Table 1 summarizes patient characteristics of cases with eICP events and controls. Decompressive craniectomy was present during a higher percentage of control records when compared to cases $(71 \%$ and $48 \%$ respectively). Etiologies of brain injury were not dissimilar, although there were slightly more case records derived from patients with $\mathrm{TBI}$, and slightly more control records derived from patients with non-traumatic hemorrhage. 
Table 1

Patient characteristics by records (cases, controls, and total).

\begin{tabular}{|c|c|c|c|}
\hline & Case $(n=21)$ & Control $(n=200)$ & Total $(n=221)$ \\
\hline Age $($ mean \pm SD) & $10 \pm 5$ & $11 \pm 5$ & $11 \pm 5$ \\
\hline Male (\%) & $8(38 \%)$ & $76(38 \%)$ & $84(38 \%)$ \\
\hline \multicolumn{4}{|l|}{ Diagnostic Category: } \\
\hline Traumatic Brain Injury & $15(71 \%)$ & $126(63 \%)$ & $141(64 \%)$ \\
\hline Non-Traumatic Hemorrhage & $4(19 \%)$ & $67(33 \%)$ & $71(32 \%)$ \\
\hline Obstructive Mass & $1(5 \%)$ & $7(3 \%)$ & $8(4 \%)$ \\
\hline Ischemic§ & $1(5 \%)$ & 0 & $1(<0.01 \%)$ \\
\hline Intraparenchymal pressure monitor (\%)* & $13(62 \%)$ & $130(63 \%)$ & $143(65 \%)$ \\
\hline Craniectomy (\%) & $10(48 \%)$ & $143(71 \%)$ & $153(69 \%)$ \\
\hline \multicolumn{4}{|l|}{ BP Medications } \\
\hline \multirow[t]{2}{*}{ Vasoactives/inotropes $(\%$, Mean VIS \pm SD) } & 6 & 32 & 38 \\
\hline & $(29 \%, 3 \pm 5)$ & $(16 \%, 1 \pm 4)$ & $(17 \%, 1 \pm 4)$ \\
\hline Anti-hypertensives & $6(29 \%)$ & $43(21 \%)$ & $49(22 \%)$ \\
\hline None & $9(43 \%)$ & $125(62 \%)$ & $134(61 \%)$ \\
\hline \multicolumn{4}{|l|}{ Pupil reactivity ${ }^{\dagger}$} \\
\hline Both & $15(71 \%)$ & $142(72 \%)$ & $157(72 \%)$ \\
\hline One & $1(5 \%)$ & $13(7 \%)$ & $14(6 \%)$ \\
\hline Neither & $5(24 \%)$ & $43(21 \%)$ & $48(22 \%)$ \\
\hline \multicolumn{4}{|c|}{ Columns contain characteristics at the time of the record. } \\
\hline \multicolumn{4}{|c|}{$\S$ Suffered a thrombotic stroke and cardiac arrest. } \\
\hline \multicolumn{4}{|c|}{$\begin{array}{l}\text { *Remainder had externalized ventricular drains (EVD). One patient had data from both an EVD and } \\
\text { intraparenchymal pressure monitor but not simultaneously. }\end{array}$} \\
\hline \multicolumn{4}{|c|}{$\begin{array}{l}\text { †Nearest to start of case or control periods. One patient did not have pupil exams documented due to } \\
\text { severe ocular/facial trauma. }\end{array}$} \\
\hline
\end{tabular}

An intraparenchymal monitor was used equally and in the majority of patients; the remainder had an externalized ventricular drain. Vasoactive support was less common during control records (16\%) compared to cases (29\%), although mean vasoactive infusion scores (VIS) were similar ( $1 \pm 4$ and $3 \pm 5$, respectively). The remaining $43 \%$ of cases and $62 \%$ of controls were not on anti-hypertensive infusions or 
vasoactive support. Pupil reactivity at the time of case and control records was similar. Deaths were distributed equally ( 1 case patient only, 1 control patient only, 1 patient contributing a case and control).

\section{Predictive modeling}

The relative performances for each of our modeling approaches at predicting elCP events with a 30 minute prediction horizon are depicted in Fig. 3. At the 30-60 minute analysis window (i.e. 30 minute prediction horizon), XGB produced a model bundle benchmark AUROC of 0.82 (95\% $\mathrm{Cl} 0.81-0.83)$. The AUPRC benchmark at the 30 minute horizon was 0.24 (95\% Cl 0.23-0.25), which was significantly above the baseline expected AUPRC of 0.10. Sensitivities of the models ranged from 0.87-0.93, with XGB achieving a sensitivity of $0.93(95 \% \mathrm{Cl} 0.91-0.95)$. Specificities ranged from $0.59-0.65$, with XGB achieving 0.65 (95\% $\mathrm{Cl} 0.64-0.66)$. Positive and negative predictive values for XGB were 0.21 and 0.99 , respectively.

\section{Feature expression in physiologic signals}

The averaged SHAP value plot depicts how the top 20 features contributed to the performance of the XBG 30-60 min predictive model bundle (Fig. 4). For instance, lower values in ICP kurtosis of the fast-Fourier transform were associated with a lower likelihood - and higher average means in the ICP were strongly associated with a higher likelihood - of developing elevated ICPs. Higher dynamics and variability in both the ICP (sample entropy) and EKG (binned entropy) signals increased likelihood of elCP events; conversely, lower dynamics and variability in DBP (mean change), SBP (standard deviation) and MAP (sample entropy) were associated with future elCP events.

Considering the 20 most contributing features in each of 10 Monte Carlo simulations with iterative splits, the heat map in Fig. 5 represents how many of these 200 features derived from each physiologic signal in every 30-minute analysis window. Said another way, this heat map conveys to what extent the top contributing features from each physiologic signal were differentially expressed.

Two results suggest the internal validity of this representation. First, differentially expressed features contained within the ICP signal increased progressively in time windows closer to eICP events. This is an expected result and can be thought of as analogous to a 'positive control' as well as to the demonstration of 'dose-response.' Second, the plethysmography oxygen saturation (Sat02) signal contributed the fewest top 20 features across all time windows - again, this was an expected result, likening it to a 'negative control.'

Strikingly, features contained within the blood pressure signal were the most differentially expressed across every time window up to 4-hours prior to eICP events, even matching ICP signal-derived features at 0-60 minutes. Features from within the blood pressure signal at $30-60$ minutes derived $40 \%, 40 \%$, and $20 \%$ from the systolic, diastolic, and mean blood pressures, respectively (not shown).

Heart rate derived features demonstrated modest differential expression. 


\section{Discussion}

The primary purpose of this pilot study was to determine whether novel physiomarkers for elCP events can be detected using machine learning methods in the physiologic data streams from continuous bedside monitors of children with severe brain injury. Secondarily, we benchmarked the predictive performance of the modeling approaches we used.

Though we expected features derived from the heart rate signal to have high differential expression because of the often-observed sympathetic surges which precede herniation events, we did not find this to be the case (Fig. 5). One reason may be because our analysis employed numerical HR series at minuteto-minute resolution and statistical feature surrogates of heart rate variability, whereas traditional heart rate variability analyses rely upon R-R interval determinations from EKG waveforms and reflect changes in autonomic state ${ }^{[20]}$. Additionally, the inherent artifact burden in the HR signal - which we did not filter - may have reduced the contributions of this signal.

We found, instead, that features contained within the blood pressure signal were consistently the most differentially expressed. This suggests that (a) physiomarkers for elCP events are concentrated within the blood pressure signal, and (b) they are accessible to machine learning analyses. The finding is consistent with the clinical observation that hemodynamic changes occur prior to eICP events, although the typical clinically observable changes in vital signs are usually coarse and very proximate to events. The strong contribution of blood pressure features to our XGB predictive model not only endured across every time window we tested, but they progressively overshadowed ICP-derived feature importance earlier in the 4hour records (Fig. 5). This was true in the context of a relatively sustained model performance across this time period (see AUPRC values). Though predictions 30 minutes prior to eICP events would enable most early interventions, the possibility of obtaining such insight hours prior to events is provocative. The notable implications of these results are that such physiomarkers could be used to predict elevated ICP in the presence of monitoring, and they may also have the potential to discriminate which children would benefit from placement of an ICP monitor.

We do not believe this result can be causatively explained by clinical differences among cases and controls. Children of different ages can have significantly disparate hemodynamic parameters, but the ages of case and control records were not different. Although $20 \%$ more control records were derived from patients who were not on either vasoactive or anti-hypertensive infusions, the mean VIS between cases and controls was similar. That said, we cannot predict how vasoactive/inotropic agents, antihypertensives, or hemodynamic-influencing sedatives impact these physiomarkers. Also, despite more control records coming from patients with craniectomies, this surgery would only serve to reduce the chances of having elCP events and not, in itself, impact hemodynamics.

The limited contribution of MAP from among the hemodynamic signals stands in interesting contrast to the findings of Güiza et al., who also reported on a machine learning model to predict elCP events using ICP and MAP as model inputs. Using 178 patients and 982 elCP events for model development, their 
model predicted elCP events 30 minutes prior to occurrence with an AUROC of 0.87 in an initial study [7] and of 0.90 when externally validated in adults ${ }^{[8]}$. With a pediatric external validation cohort, these authors found a lower AUROC of 0.79 , comparable to our benchmark AUROC of 0.82 . This may have been because their development cohort contained only 13 pediatric patients. Sensitivities were similar ( $92 \%$ and $93 \%$ ), though our model achieved better specificity (48\% vs 65\%). Güiza et al.'s excellent predictive performance using MAP and ICP alone may be a reflection of their large training cohorts as well as their predominantly adult population which has less vital sign variation than children. It is also possible that different machine learning modeling approaches - and importantly, the features which are examined may modulate the measured contribution of each physiologic signal. Nevertheless, our findings that differentially expressed physiomarkers are concentrated within the blood pressure signal as well as the ICP signal are consistent with Güiza et al.'s modeling results.

Though our exclusively pediatric cohort of different ages and mixed brain injury pathologies who underwent standard brain injury management may make the results of our small pilot study generalizable, they require externally validation in a larger cohort. There are several limitations. Our sample size was small for machine learning. Though minute-to-minute data resolution may be accessible to more ICUs, it may have also constrained our model performance. Measures of change and variability were limited in our feature selection, and our design did not consider changes in feature values over time across analysis windows. Our records were curated and imbalanced. We did not study the impact of vasoactive agents or sedatives (e.g. propofol, dexmedetomidine) which may have hemodynamic effects, or of management conditions (e.g. craniectomy, type of ICP monitor, etc.), though their use appear similar across cases and controls.

In summary, we find that discriminatory physiomarkers discernable by machine learning methods are concentrated within blood pressure data for up to 4 hours prior to future elCP events in children with severe brain injuries, and models using them demonstrate strong benchmark performance. Taken in aggregate, our findings support the idea that future attempts at high fidelity predictive modeling of elCP events should leverage features contained within hemodynamic signals.

\section{Declarations}

\section{Author contributions statement}

Kassi Ackerman and Akram Mohammed contributed equally to this work. Kassi Ackerman is responsible for study design, IRB approval, clinical data extraction, and manuscript writing. Akram Mohammed is responsible for coding, machine learning modeling, and manuscript writing. Lokesh Chinthala contributed by data extraction and preprocessing. Robert Davis and Rishikesan Kamaleswaran contributed to study design and model analyses. Nadeem I. Shafi conceived the project and contributed heavily to approach, design, manuscript writing and revisions.

\section{References}


1. Fodstad, H., Kelly, P. J. \& Buchfelder, M. History of the Cushing reflex. Neurosurgery vol. 59(2006).

2. Grady, P. A. \& Blaumanis, O. R. Physiologic parameters of the cushing reflex.Surg. Neurol.29, (1988).

3. Stein, D. M. et al. Brief episodes of intracranial hypertension and cerebral hypoperfusion are associated with poor functional outcome after severe traumatic brain injury.J. Trauma - Inj. Infect. Crit. Care71, (2011).

4. Miller Ferguson, N. et al. Intracranial Hypertension and Cerebral Hypoperfusion in Children With Severe Traumatic Brain Injury. Pediatr. Crit. Care Med, 17, 444-450 (2016).

5. Kochanek, P. M. et al. Guidelines for the Management of Pediatric Severe Traumatic Brain Injury, Third Edition: Update of the Brain Trauma Foundation Guidelines, Executive Summary.Clin. Neurosurg.84, (2019).

6. Allen, B. B., Chiu, Y. L., Gerber, L. M., Ghajar, J. \& Greenfield, J. P. Age-specific cerebral perfusion pressure thresholds and survival in children and adolescents with severe traumatic brain injury. Pediatr. Crit. Care Med, 15, 62-70 (2014).

7. Güiza, F., Depreitere, B., Piper, I., Van den Berghe, G. \& Meyfroidt, G. Novel Methods to Predict Increased Intracranial Pressure During Intensive Care and Long-Term Neurologic Outcome After Traumatic Brain Injury. Crit. Care Med, 41, 554-564 (2013).

8. Güiza, F. et al. Early Detection of Increased Intracranial Pressure Episodes in Traumatic Brain Injury. Crit. Care Med, 45, e316-e320 (2017).

9. Griffin, M. P. et al. Heart rate characteristics: Novel physiomarkers to predict neonatal infection and death.Pediatrics116, (2005).

10. Alqahtani, M. F., Marsillio, L. E. \& Rozenfeld, R. A. A review of biomarkers and physiomarkers in pediatric sepsis.Clin. Pediatr. Emerg. Med.15, (2014).

11. Kamaleswaran, R. et al. Applying artificial intelligence to identify physiomarkers predicting severe sepsis in the PICU.Pediatr. Crit. Care Med.19, (2018).

12. Rajkomar, A., Dean, J. \& Kohane, I. Machine Learning in Medicine.N. Engl. J. Med.380, (2019).

13. Cabitza, F., Rasoini, R. \& Gensini, G. F. Unintended consequences of machine learning in medicine.JAMA - Journal of the American Medical Associationvol.318(2017).

14. Reyna, M. A. et al. Early prediction of sepsis from clinical data: The PhysioNet/computing in cardiology challenge 2019. Crit. Care Med, https://doi.org/10.1097/CCM.0000000000004145 (2020).

15. Thrall, J. H. et al. Artificial Intelligence and Machine Learning in Radiology: Opportunities, Challenges, Pitfalls, and Criteria for Success.J. Am. Coll. Radiol.15, (2018).

16. Vaswani, A. et al. Attention is all you need. in Advances in Neural Information Processing Systems vols 2017-December(2017).

17. Kubota, K. J., Chen, J. A. \& Little, M. A. Machine learning for large-scale wearable sensor data in Parkinson's disease: Concepts, promises, pitfalls, and futures.Mov. Disord.31, (2016).

18. Snoek, J., Larochelle, H. \& Adams, R. P. Practical Bayesian optimization of machine learning algorithms. inAdvances in Neural Information Processing Systemsvol. 4 (2012). 
19. Lundberg, S. M. \& Lee, S. I. A unified approach to interpreting model predictions. in Advances in Neural Information Processing Systems vols 2017-December(2017).

20. Malik, M. et al. Heart rate variability. Standards of measurement, physiological interpretation, and clinical use.European Heart Journalvol. 17 (1996).

\section{Figures}

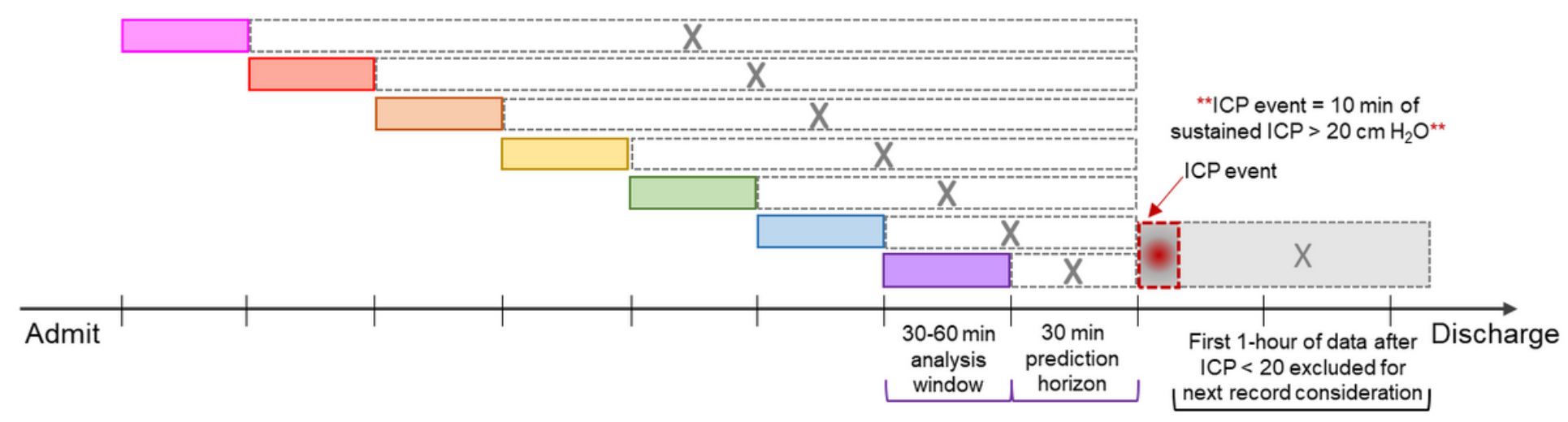

\section{Figure 1}

eICP Event Schematic. The prediction horizon is the time prior to the ICP event over which features from the preceding analysis window are used for prediction. ICP had to remain $<20 \mathrm{~cm} \mathrm{H} 20$ for one hour before subsequent data could be considered for inclusion. 


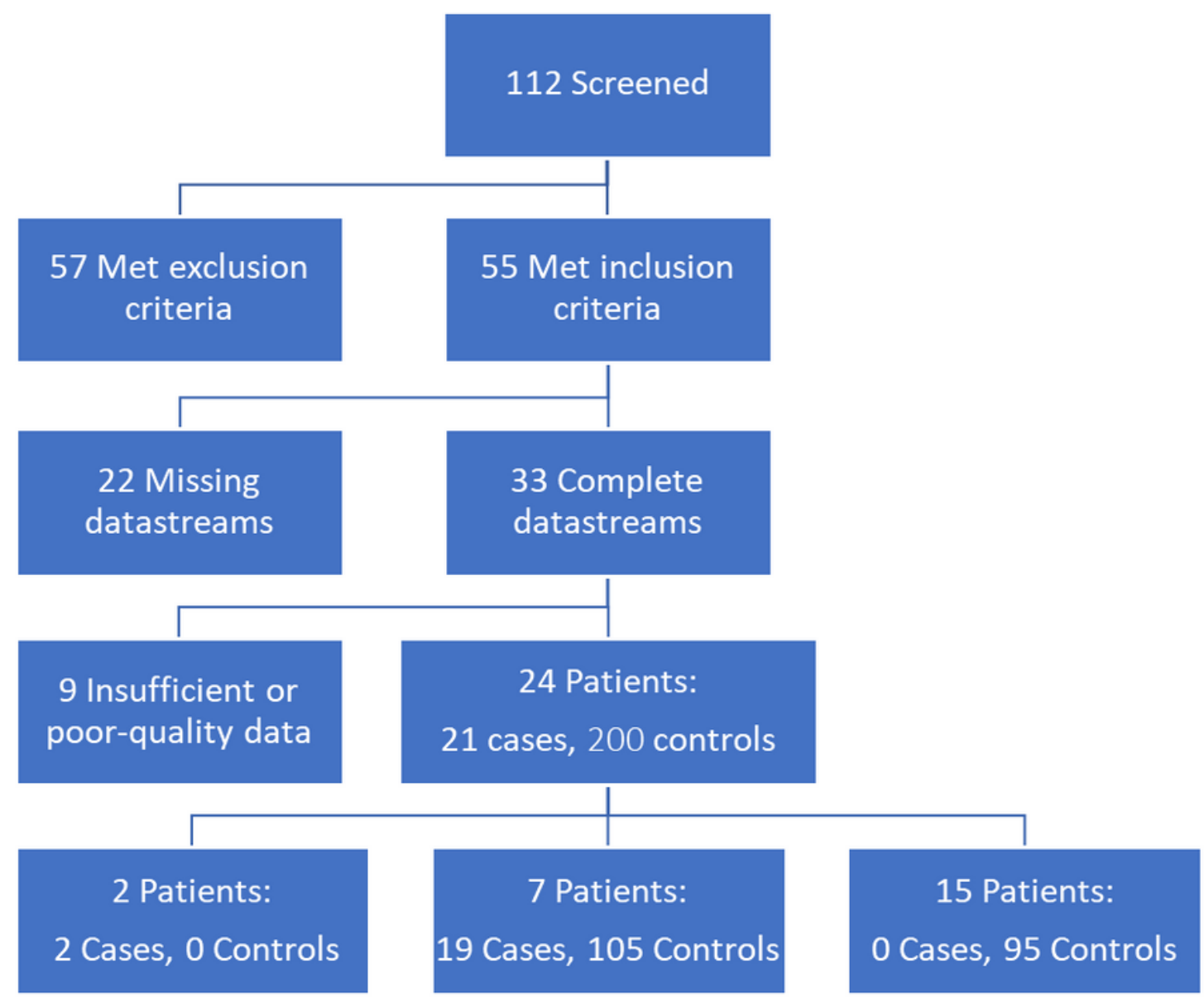

Figure 2

Consort Diagram. Data was regarded as "insufficient" if $>20 \min (\sim 10 \%$ of a record) was poor quality or missing. 


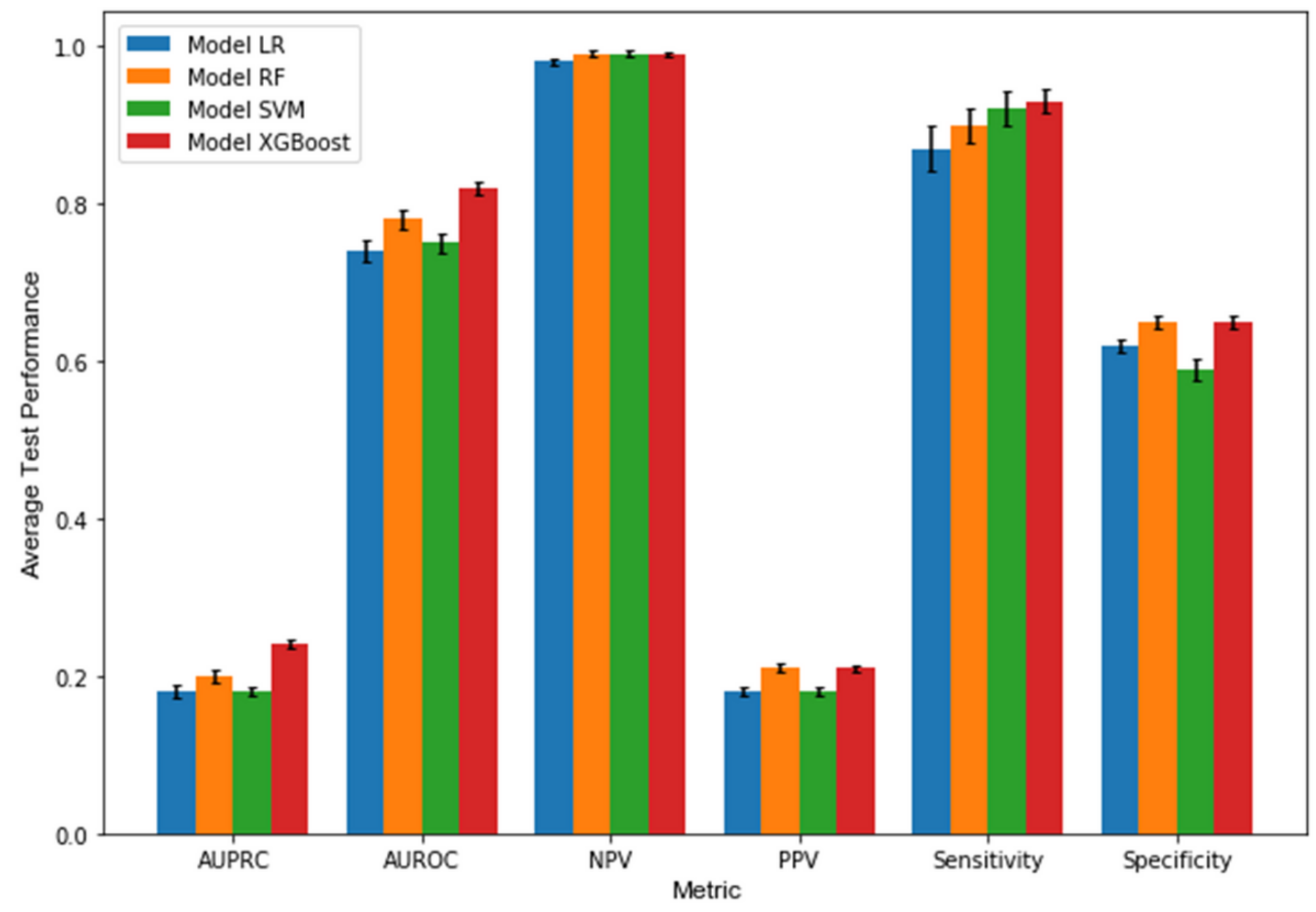

Figure 3

Performance of each modeling approach for predicting eICP events with a 30 minute prediction horizon. Error bars $=95 \%$ confidence intervals. 
ICP_fft_aggregated_aggtype_"kurtosis" ICP_mean ICP_sample_entropy ICP_mean_abs_change DBP_mean_change EKG_HR_binned_entropy_max_bins_10 SBP_standard_deviation CPP_binned_entropy_max_bins_10 PulseRate_maximum ICP_fft_aggregated_aggtype_"skew" PulseRate_binned_entropy_max_bins_10 SatO2_fft_aggregated_aggtype_"variance" EKG_HR_fft_aggregated_aggtype_"kurtosis" PulseRate_mean_change MAP_mean_abs_change ICP_mean_change MAP_sample_entropy EKG_HR_standard_deviation ICP_fft_aggregated_aggtype_"variance" DBP_binned_entropy_max_bins_10

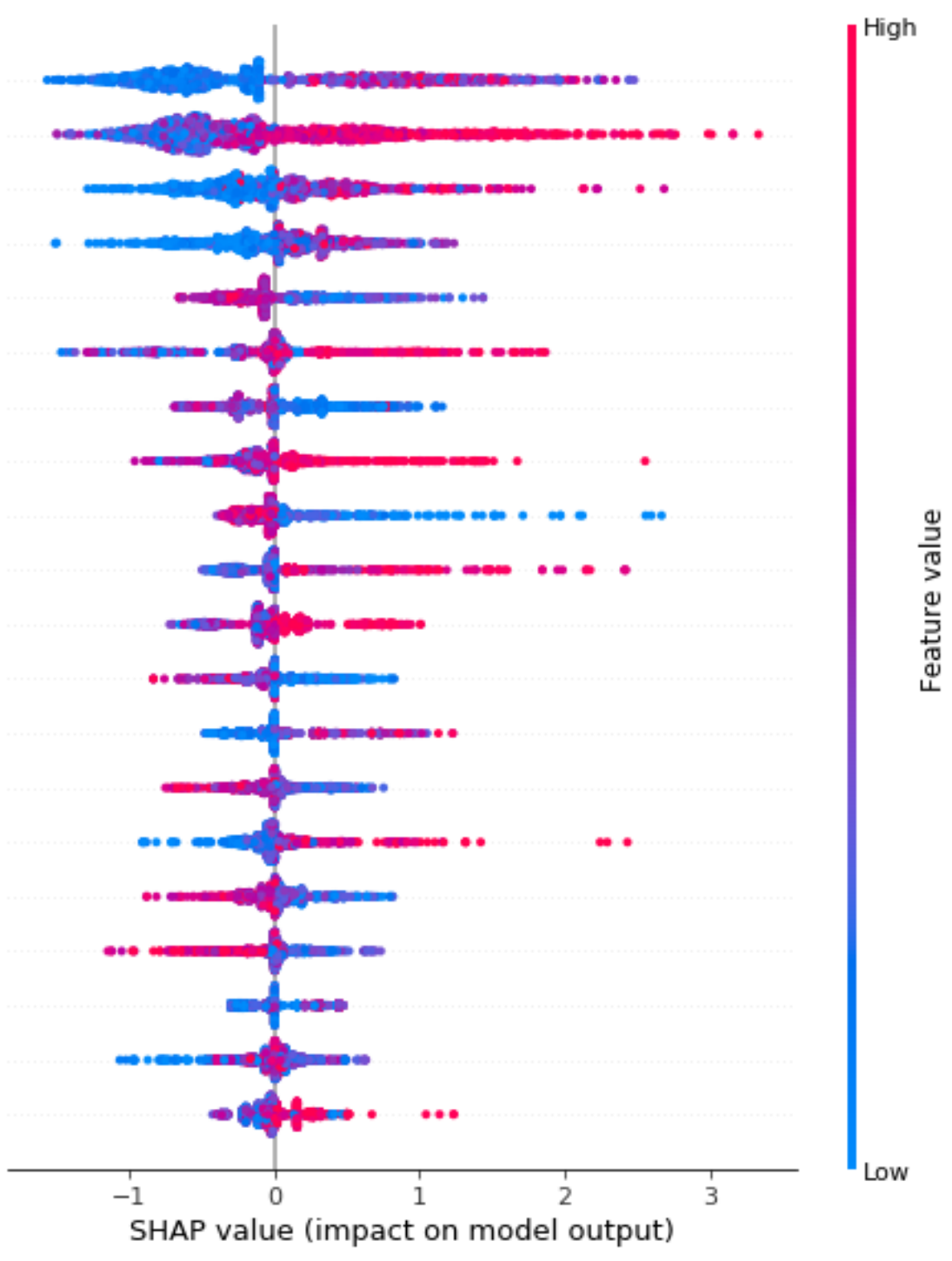

\section{Figure 4}

Average SHAP value plot. Displayed in descending order are the 20 most important features contributing to the XGB model bundle performance with the 30-minute prediction horizon, along with their averaged SHAP values. 


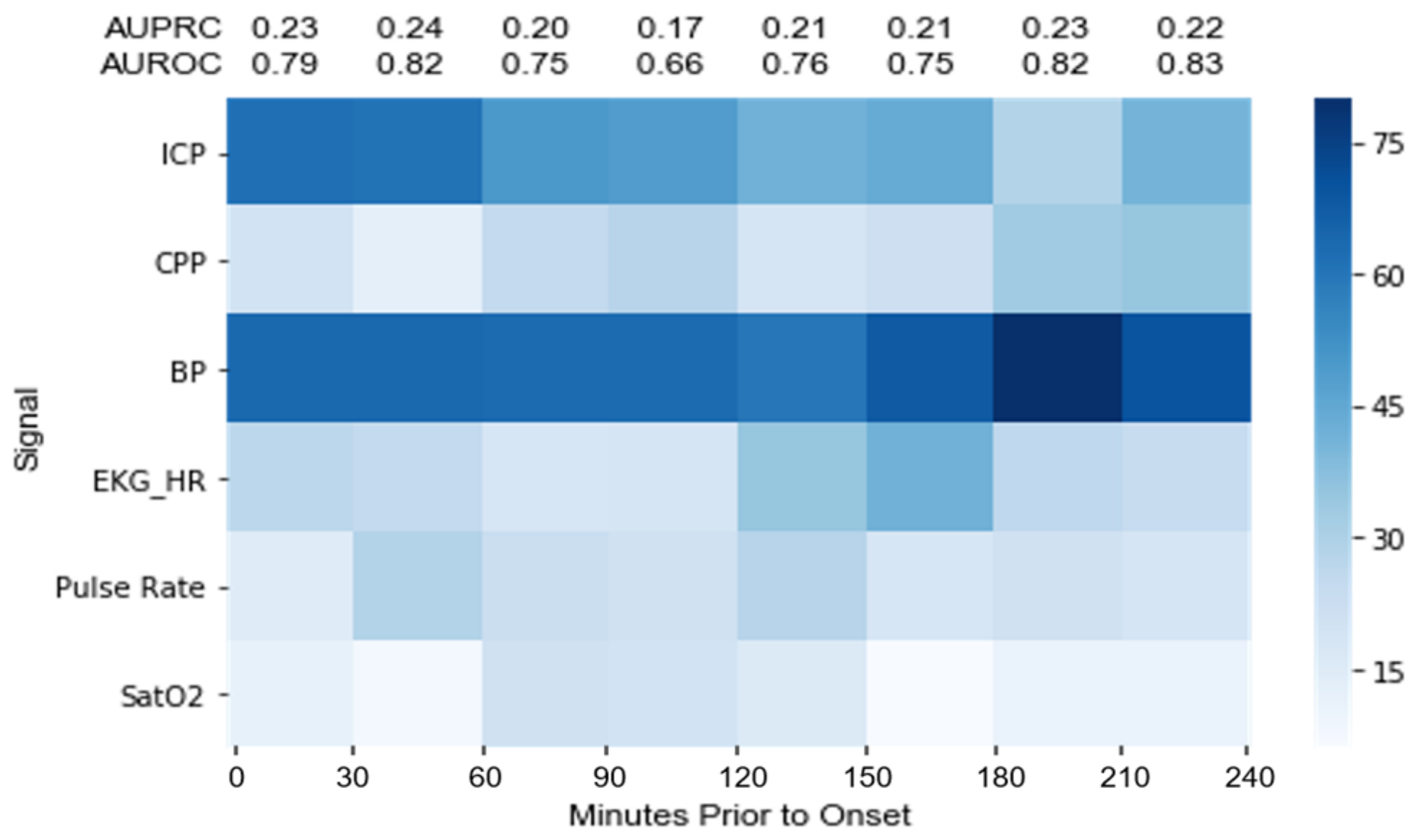

Figure 5

Heat map of most important features across 10 training-testing iterations in every 30 minute analysis window. 200 features in each column are categorized by physiologic signal. Darker blue means a greater number of features derived from the physiologic signal.

\section{Supplementary Files}

This is a list of supplementary files associated with this preprint. Click to download.

- Supplementaryfigures.pdf 